\title{
Two Expanding Integrable Models of the Geng-Cao Hierarchy
}

\author{
Xiurong Guo, ${ }^{1}$ Yufeng Zhang, ${ }^{2}$ and Xuping Zhang ${ }^{1}$ \\ ${ }^{1}$ Department of Basic Courses, Shandong University of Science and Technology, Tai'an, Shandong 271019, China \\ ${ }^{2}$ College of Science, China University of Mining and Technology, Xuzhou 221116, China \\ Correspondence should be addressed to Xiurong Guo; springming007@126.com
}

Received 21 November 2013; Accepted 13 January 2014; Published 27 February 2014

Academic Editor: Tiecheng Xia

Copyright (C) 2014 Xiurong Guo et al. This is an open access article distributed under the Creative Commons Attribution License, which permits unrestricted use, distribution, and reproduction in any medium, provided the original work is properly cited.

\begin{abstract}
As far as linear integrable couplings are concerned, one has obtained some rich and interesting results. In the paper, we will deduce two kinds of expanding integrable models of the Geng-Cao (GC) hierarchy by constructing different 6-dimensional Lie algebras. One expanding integrable model (actually, it is a nonlinear integrable coupling) reduces to a generalized Burgers equation and further reduces to the heat equation whose expanding nonlinear integrable model is generated. Another one is an expanding integrable model which is different from the first one. Finally, the Hamiltonian structures of the two expanding integrable models are obtained by employing the variational identity and the trace identity, respectively.
\end{abstract}

\section{Introduction}

Integrable couplings are a kind of expanding integrable models of some known integrable hierarchies of equations. Based on this theory, one has obtained some integrable couplings of the known integrable hierarchies [1-8]. These integrable couplings are all linear with respect to the coupled variables. That is, if we introduce an evolution equation $U_{t}=$ $K(u)$, the coupled variable $V$ satisfying $V_{t}=S(u, v)$ is linear in $V$. The reason for this may be given by special Lie algebras. That is, such a Lie algebra $G$ can be decomposed into a sum of the two subalgebras $G_{1}$ and $G_{2}$, which meets

$$
G=G_{1} \oplus G_{2}, \quad\left[G_{1}, G_{2}\right] \subset G_{2} .
$$

If the subalgebra $G_{2}$ is not simple, then the integrable coupling

$$
\begin{aligned}
U_{t} & =K(u), \\
V_{t} & =S(u, v)
\end{aligned}
$$

is linear with respect to the variable $V$, which is obtained by introducing Lax pairs through the Lie algebra $G$. However, it is more interesting to seek for nonlinear integrable couplings because most of the coupled dynamics from physics, mechanics, and so forth are nonlinear. Recently, Ma and Zhu [9] introduced a kind of Lie algebra to deduce the nonlinear integrable couplings of the nonlinear Schrödinger equation and so forth, where the Lie subalgebras are simple and are different from the above. Based on this, Zhang [10] proposed a simple and efficient method for generating nonlinear integrable couplings and obtained the nonlinear integrable couplings of the Giachetti-Johnson (GJ) hierarchy and the Yang hierarchy, respectively. In addition, Zhang and Hon [11] proposed another Lie algebra which is different from those in $[9,10]$ to deduce nonlinear integrable couplings. Wei and Xia [12] also obtained some nonlinear integrable couplings of the known integrable hierarchies.

In the paper, we want to start from a spectral problem proposed by Geng and Cao [13] to deduce an integrable hierarchy (called the GC hierarchy) under the frame of zero curvature equations by the Tu scheme [14] and obtain its new Hamiltonian structure. Then with the help of a 6-dimensional Lie algebra, a nonlinear expanding integrable model of the GC hierarchy is obtained, whose Hamiltonian structure is generated by the variational identity presented in [15]. The expanding integrable model can reduce to a generalized Burgers equation and further reduce to the heat equation. Another new 6-dimensional Lie algebra is constructed for which the second expanding integrable model is produced by using the Tu scheme whose Hamiltonian structure is derived from the trace identity proposed by $\mathrm{Tu}$ [14]. We shall find the two expanding integrable models of the GC hierarchy are different. 


\section{The GC Integrable Hierarchy and Its Hamiltonian Structure}

We have known that

$$
h=\left(\begin{array}{cc}
1 & 0 \\
0 & -1
\end{array}\right), \quad e=\left(\begin{array}{ll}
0 & 1 \\
0 & 0
\end{array}\right), \quad f=\left(\begin{array}{ll}
0 & 0 \\
1 & 0
\end{array}\right),
$$

then one gets

$$
[h, e]=2 e, \quad[h, f]=-2 f, \quad[e, f]=h .
$$

It is well known that $\operatorname{span}\{h, e, f\}=A_{1}$ is a Lie algebra. A loop algebra of $A_{1}$ is given by

$$
\widetilde{A_{1}}=\operatorname{span}\{h(n), e(n), f(n)\},
$$

where

$$
\begin{gathered}
h(n)=h(0) \lambda^{n}, \quad e(n)=e(0) \lambda^{n}, \\
h(n)=h(0) \lambda^{n}, \\
n \in Z .
\end{gathered}
$$

By using the loop algebra $\widetilde{A_{1}}$, introduce an isospectral problem [13]:

$$
U=\left(\begin{array}{cc}
-\lambda & \lambda u \\
v & \lambda
\end{array}\right)=-h(1)+u e(1)+v f(0) .
$$

Set

$$
V=V_{1} h(0)+V_{2} e(0)+V_{3} f(0)
$$

where

$$
V_{i}=\sum_{m \geq 0} V_{i m} h(-m), \quad i=1,2,3
$$

The stationary equation $V_{x}=[U, V]$ admits that a solution for the $V$ is as follows:

$$
\begin{gathered}
\left(V_{1 m}\right)_{x}=u V_{3 m}-v V_{2 m}, \\
\left(V_{2 m}\right)_{x}=-2 V_{2, m+1}-2 u V_{1, m+1}, \\
\left(V_{3 m}\right)_{x}=2 V_{3, m+1}+2 v V_{1, m+1},
\end{gathered}
$$

which gives rise to

$$
\left(V_{1, m+1}\right)_{x}=\frac{1}{2} u\left(V_{3 m}\right)_{x}-\frac{1}{2} v\left(V_{2 m}\right)_{x}
$$

Set

$$
V_{1,0}=V_{2,0}=V_{3,0}=0, \quad V_{1,1}=\alpha ;
$$

from (10) and (11) we have

$$
\begin{gathered}
V_{2,1}=-\alpha u, \quad V_{3,1}=-\alpha v, \quad V_{1,2}=-\frac{\alpha}{2} u v, \\
V_{2,2}=\frac{\alpha}{2}\left(u_{x}+u^{2} v\right), \quad V_{3,2}=\frac{\alpha}{2}\left(-v_{x}+u v^{2}\right), \ldots
\end{gathered}
$$

denote by

$$
V^{(n)}=\sum_{m=0}^{n}\left(V_{1 m} h(n-m)+V_{2 m} e(n-m)+V_{3 m} f(n-m)\right) \text {. }
$$

We have

$$
\begin{aligned}
-V_{x}^{(n)}+\left[U, V^{(n)}\right] & =\left(2 V_{2, n+1}+2 u V_{1, n+1}\right) e(1) \\
& -2\left(V_{3, n+1}+2 V_{1, n+1}\right) f(0) .
\end{aligned}
$$

The compatibility of the following Lax pair

$$
U=-h(1)+u e(1)+v f(0)
$$

$$
V^{(n)}=\sum_{m=0}^{n}\left(V_{1 m} h(n-m)+V_{2 m} e(n-m)+V_{3 m} f(n-m)\right)
$$

gives rise to

$$
\begin{aligned}
\left(\begin{array}{l}
u \\
v
\end{array}\right)_{t_{n}} & =\left(\begin{array}{c}
-2 V_{2, n+1}-2 u V_{1, n+1} \\
2 V_{3, n+1}+2 v V_{1, n+1}
\end{array}\right)=\left(\begin{array}{c}
\left(V_{2 n}\right)_{x} \\
\left(V_{3 n}\right)_{x}
\end{array}\right) \\
& =\left(\begin{array}{ll}
0 & \partial \\
\partial & 0
\end{array}\right)\left(\begin{array}{l}
V_{3 n} \\
V_{2 n}
\end{array}\right)=J\left(\begin{array}{l}
V_{3 n} \\
V_{2 n}
\end{array}\right),
\end{aligned}
$$

where

$$
J=\left(\begin{array}{ll}
0 & \partial \\
\partial & 0
\end{array}\right)
$$

is a Hamiltonian operator.

By the trace identity presented in [14], we have

$$
\begin{gathered}
\left\langle V, \frac{\partial U}{\partial u}\right\rangle=\lambda V_{3}, \quad\left\langle V, \frac{\partial U}{\partial v}\right\rangle=\lambda V_{2}, \\
\left\langle V, \frac{\partial U}{\partial \lambda}\right\rangle=-2 \lambda V_{1}+u V_{3} .
\end{gathered}
$$

Substituting the above results to the trace identity yields

$$
\frac{\delta}{\delta w}\left(-2 \lambda V_{1}+u V_{3}\right)=\lambda^{-\gamma} \frac{\partial}{\partial \lambda} \lambda^{\gamma}\left(\begin{array}{l}
\lambda V_{3} \\
\lambda V_{2}
\end{array}\right)
$$

where

$$
\frac{\delta}{\delta w}=\left(\frac{\delta}{\delta u}, \frac{\delta}{\delta v}\right)^{T}
$$

Comparing the coefficients of $\lambda^{-n}$ of both sides in (20) gives

$$
\frac{\delta}{\delta w}\left(-2 V_{1, n+1}+u V_{3, n+1}\right)=(-n+1+\gamma)\left(\begin{array}{l}
\lambda V_{3 n} \\
\lambda V_{2 n}
\end{array}\right)
$$

It is easy to see $\gamma=-1$. Thus, we have

$$
\left(\begin{array}{l}
\lambda V_{3 n} \\
\lambda V_{2 n}
\end{array}\right)=\frac{\delta}{\delta u}\left(\frac{2 V_{1, n+1}-u V_{3, n+1}}{n}\right)=\frac{\delta H_{n}}{\delta u},
$$


where

$$
H_{n}=\frac{2 V_{1, n+1}-u V_{3, n+1}}{n}
$$

are Hamiltonian conserved densities of the Lax integrable hierarchy (17). Therefore, we get a Hamiltonian form of the hierarchy (17) as follows:

$$
\left(\begin{array}{l}
u \\
v
\end{array}\right)_{t_{n}}=J \frac{\delta H_{n}}{\delta u} .
$$

Let us consider the reduced cases of (17). When $n=1$, we get that

$$
u_{t_{1}}=-\alpha u_{x}, \quad v_{t_{1}}=-\alpha v_{x} .
$$

Taking $n=2$, one gets a generalized Burgers equation:

$$
\begin{aligned}
& u_{t_{2}}=\frac{\alpha}{2} u_{x x}+\alpha u u_{x} v+\frac{\alpha}{2} u^{2} v_{x}, \\
& v_{t_{2}}=-\frac{\alpha}{2} v_{x x}+\frac{\alpha}{2} u_{x} v^{2}+\alpha u v v_{x} .
\end{aligned}
$$

Remark 1. The Hamiltonian structure (23) is different from that in [14]. We call (17) the GC hierarchy.

\section{The First Expanding Integrable Model of the GC Hierarchy}

Zhang and Tam [16] proposed a few kinds of Lie algebras to deduce nonlinear integrable couplings. In the section we will choose one of them to investigate the nonlinear integrable coupling of the hierarchy (17).

Consider the following Lie algebra:

$$
F=\operatorname{span}\left\{f_{1}, \ldots, f_{6}\right\},
$$

where

$$
\begin{array}{clc}
f_{1}=\left(\begin{array}{cc}
e_{1} & 0 \\
0 & e_{1}
\end{array}\right), & f_{2}=\left(\begin{array}{cc}
e_{2} & 0 \\
0 & e_{2}
\end{array}\right), & f_{3}=\left(\begin{array}{cc}
e_{3} & 0 \\
0 & e_{3}
\end{array}\right), \\
f_{4}=\left(\begin{array}{ll}
0 & e_{1} \\
0 & e_{1}
\end{array}\right), & f_{5}=\left(\begin{array}{ll}
0 & e_{2} \\
0 & e_{2}
\end{array}\right), & f_{6}=\left(\begin{array}{ll}
0 & e_{3} \\
0 & e_{3}
\end{array}\right), \\
e_{1}=\left(\begin{array}{cc}
1 & 0 \\
0 & -1
\end{array}\right), & e_{2}=\left(\begin{array}{ll}
0 & 1 \\
0 & 0
\end{array}\right), & e_{3}=\left(\begin{array}{ll}
0 & 0 \\
1 & 0
\end{array}\right) .
\end{array}
$$

Define

$$
[a, b]=a b-b a, \quad \forall a, b \in F .
$$

It is easy to compute that

$$
\begin{gathered}
{\left[f_{1}, f_{2}\right]=2 f_{2}, \quad\left[f_{1}, f_{3}\right]=-2 f_{3}, \quad\left[f_{2}, f_{3}\right]=f_{1},} \\
{\left[f_{1}, f_{4}\right]=0, \quad\left[f_{1}, f_{5}\right]=2 f_{5},} \\
{\left[f_{1}, f_{6}\right]=-2 f_{6}, \quad\left[f_{2}, f_{4}\right]=-2 f_{5}, \quad\left[f_{2}, f_{5}\right]=0,} \\
{\left[f_{2}, f_{6}\right]=f_{4}, \quad\left[f_{3}, f_{4}\right]=2 f_{6},} \\
{\left[f_{3}, f_{5}\right]=-f_{4}, \quad\left[f_{3}, f_{6}\right]=0, \quad\left[f_{4}, f_{5}\right]=2 f_{5},} \\
{\left[f_{4}, f_{6}\right]=-2 f_{6}, \quad\left[f_{5}, f_{6}\right]=f_{4} .}
\end{gathered}
$$

Set

$$
F_{1}=\operatorname{span}\left\{f_{1}, f_{2}, f_{3}\right\}, \quad F_{2}=\operatorname{span}\left\{f_{4}, f_{5}, f_{6}\right\} ;
$$

we have

$$
F_{1}=F_{1} \oplus F_{2}, \quad\left[F_{1}, F_{2}\right] \subset F_{2}
$$

$F_{1}$ and $F_{2}$ are all simple Lie-subalgebras of the Lie algebra $F$. The corresponding symmetric constant matrix $M$ appearing in the variational identity is that

$$
\left(\begin{array}{cccccc}
2 \eta_{1} & 0 & 0 & 2 \eta_{2} & 0 & 0 \\
0 & 0 & \eta_{1} & 0 & 0 & \eta_{2} \\
0 & \eta_{1} & 0 & 0 & \eta_{2} & 0 \\
2 \eta_{2} & 0 & 0 & 2 \eta_{2} & 0 & 0 \\
0 & 0 & \eta_{2} & 0 & 0 & \eta_{2} \\
0 & \eta_{2} & 0 & 0 & \eta_{2} & 0
\end{array}\right) .
$$

A loop algebra corresponding to the Lie algebra $F$ is defined by

$$
\begin{gathered}
\widetilde{F}=\operatorname{span}\left\{f_{1}(n), \ldots, f_{6}(n)\right\}, \quad f_{i}(n)=f_{i} \lambda^{n}, \\
{\left[f_{i}(m), f_{j}(n)\right]=\left[f_{i}, f_{j}\right] \lambda^{m+n}, \quad 1 \leq i, j \leq 6, m, n \in Z .}
\end{gathered}
$$

We use the loop algebra $\widetilde{F}$ to introduce a Lax pair:

$$
\begin{gathered}
U=-f(1)+u f_{2}(1)+v f_{3}(0)+u_{1} f_{5}(1)+u_{2} f_{6}(0), \\
V=\sum_{m \geq 0}\left(V_{1 m} f_{1}(1-m)+V_{2 m} f_{2}(1-m)+V_{3 m} f_{3}(-m)\right. \\
\left.\quad+V_{4 m} f_{4}(1-m)+V_{5 m} f_{5}(1-m)+V_{6 m} f_{6}(-m)\right) .
\end{gathered}
$$

The stationary equation $V_{x}=[U, V]$ is equivalent to

$$
\begin{gathered}
\left(V_{1 m}\right)_{x}=u V_{3 m}-v V_{2 m}, \\
\left(V_{2 m}\right)_{x}=-2 V_{2, m+1}-2 u V_{1, m+1}, \\
\left(V_{3 m}\right)_{x}=2 V_{3, m+1}+2 v V_{1, m+1}, \\
\left(V_{4 m}\right)_{x}=u_{1} V_{3 m}-u_{2} V_{2 m}-\left(v+u_{2}\right) V_{5 m}+\left(u+u_{1}\right) V_{6 m}, \\
\left(V_{5 m}\right)_{x}=-2 V_{5, m+1}-2 u_{1} V_{1, m+1}-2\left(u+u_{1}\right) V_{4, m+1}, \\
\left(V_{6 m}\right)_{x}=2 V_{6, m+1}+2 u_{2} V_{1, m+1}+2\left(v+u_{2}\right) V_{4, m+1}
\end{gathered}
$$

from which we have

$$
\begin{aligned}
&\left(V_{1, m+1}\right)_{x}=\frac{1}{2} u\left(V_{3 m}\right)_{x}-\frac{1}{2} v\left(V_{2 m}\right)_{x} \\
&\left(V_{4, m+1}\right)= \frac{1}{2} u_{1}\left(V_{3 m}\right)_{x}+\frac{1}{2} u_{2}\left(V_{2 m}\right)_{x} \\
&+\frac{1}{2}\left(u+u_{2}\right)\left(V_{5 m}\right)_{x}+\frac{1}{2}\left(u+u_{1}\right)\left(V_{6 m}\right)_{x} .
\end{aligned}
$$


Set

$$
V_{1,0}=V_{2,0}=V_{3,0}=V_{4,0}=V_{5,0}=V_{6,0}=0, \quad V_{1,1}=\alpha ;
$$

we obtain from (37)

$$
\begin{gathered}
V_{3,1}=-\alpha v, \quad V_{2,1}=-\alpha u, \quad V_{4,1}=0, \\
V_{4,1}=-\alpha u_{1}, \quad V_{6,1}=-\alpha u_{2}, \\
V_{1,2}=-\frac{\alpha}{2} u v, \quad V_{4,2}=-\frac{\alpha}{2}\left(u_{1} v+u v_{2}+u_{1} u_{2}\right), \\
V_{5,2}=\frac{\alpha}{2}\left(u_{1} x+u_{1} u v+\left(u+u_{1}\right)\left(u_{1} v+u u_{2}+u_{1} u_{2}\right)\right), \\
V_{6,2}=\frac{\alpha}{2}\left(-u_{2} x+u_{2} u v+\left(v+u_{2}\right)\left(u_{1} v+u u_{2}+u_{1} u_{2}\right)\right), \ldots
\end{gathered}
$$

Note

$$
\begin{aligned}
V^{(n)}=\sum_{m=0}^{n}( & V_{1 m} f_{1}(1+n-m)+V_{2 m} f_{2}(1+n-m) \\
& +V_{3 m} f_{3}(-m)+V_{4 m} f_{4}(1+n-m) \\
& \left.+V_{5 m} f_{5}(1+n-m)+V_{6 m} f_{6}(n-m)\right) ;
\end{aligned}
$$

a direct calculation yields

$$
\begin{aligned}
-V_{x}^{(n)}+ & {\left[U, V^{(n)}\right] } \\
= & -2\left(V_{3, n+1}+v V_{1, n+1}\right) f_{3}(0) \\
& +\left(2 V_{2, n+1}+2 u V_{1, n+1}\right) f_{2}(1) \\
& -2\left(\left(v+u_{2}\right) V_{4, n+1}+u_{2}\right) V_{1, n+1}+V_{6, n+1} f_{6}(0) \\
& +2\left(\left(u+u_{1}\right) V_{4, n+1}+u_{1}\right) V_{1, n+1}+V_{5, n+1} f_{5}(1) \\
= & -\left(V_{3 n}\right)_{x} f_{3}(0)-\left(V_{2 n}\right)_{x} f_{2}(1) \\
& -\left(V_{6 n}\right)_{x} f_{6}(0)-\left(V_{5 n}\right)_{x} f_{5}(1) .
\end{aligned}
$$

Therefore, zero curvature equation

$$
U_{t}-V_{x}^{n}+\left[U, V^{(n)}\right]=0
$$

admits that

$$
\left(\begin{array}{c}
u \\
v \\
u_{1} \\
u_{2}
\end{array}\right)=\left(\begin{array}{l}
\left(V_{2 n}\right)_{x} \\
\left(V_{3 n}\right)_{x} \\
\left(V_{5 n}\right)_{x} \\
\left(V_{6 n}\right)_{x}
\end{array}\right) .
$$

Set $u_{1}=u_{2}=0,(44)$ reduces to the integrable hierarchy (17). When we take $n=2$, we get an expanding nonlinear integrable model of the generalized Burgers equation (27) as follows:

$$
\begin{gathered}
u_{t_{2}}=\frac{\alpha}{2} u_{x x}+\alpha u u_{x} v+\frac{\alpha}{2} u^{2} v_{x}, \\
v_{t_{2}}=-\frac{\alpha}{2} v_{x x}+\frac{\alpha}{2} u_{x} v^{2}+\alpha u v v_{x}, \\
u_{1 t_{2}=}=\frac{\alpha}{2}\left(u_{1 x x}+u_{1 x} u v+u_{1}(u v)_{x}\right. \\
+\left(u+u_{1}\right)_{x}\left(u_{1} v+u u_{2}+u_{1} u_{2}\right) \\
\left.+\left(u+u_{1}\right)\left(u_{1} v+u u_{2}+u_{1} u_{2}\right)_{x}\right), \\
u_{2 t_{2}=\frac{\alpha}{2}}\left(-u_{2 x x}+u_{2 x} u v+u_{2}(u v)_{x}\right. \\
+\left(v+u_{2}\right)_{x}\left(u_{1} v+u u_{2}+u_{1} u_{2}\right) \\
\left.+\left(v+u_{2}\right)\left(u_{1} v+u u_{2}+u_{1} u_{2}\right)_{x}\right) .
\end{gathered}
$$

Obviously, the coupled equations are nonlinear with respect to the coupled variables $u_{1}$ and $u_{2}$. Therefore, the hierarchy (44) is a nonlinear expanding integrable model of the integrable system (17); actually, it is a nonlinear integrable coupling.

The nonlinear expanding integrable model (45) can be written as two parts, one is just right (27); another one is the latter two equations in (45), which can be regarded as a coupled nonlinear equation with variable coefficients $u, v$, and their derivatives in the variable $x$, where the functions $u$, $v$ satisfy (27). In particular, we take a trivial solution of (27) to be $u=v=0$; then (45) reduces to the following equations:

$$
\begin{aligned}
& u_{1 t_{2}}=\frac{\alpha}{2}\left[u_{1, x x}+\left(u_{1}^{2} u_{2}\right)_{x}\right], \\
& u_{2 t_{2}}=\frac{\alpha}{2}\left[-u_{2, x x}+\left(u_{2}^{2} u_{1}\right)_{x}\right] .
\end{aligned}
$$

When we set $u_{2}=0$, the above equations reduce to the wellknown heat equation.

In order to deduce Hamiltonian structure of the nonlinear integrable coupling (44), we define a linear functional [11]:

$$
\{a, b\}=a^{T} M b,
$$

where $a=\left(a_{1}, \ldots, a_{6}\right)^{T}, b=\left(b_{1}, \ldots, b_{6}\right)^{T}$.

It is easy to see that the Lie algebra $F$ is isomorphic to the Lie algebra $R^{6}$ if equipped with a commutator as follows:

$$
\begin{aligned}
{[a, b]^{T}=\left(a_{2} b_{3}-a_{3} b_{2}, 2 a_{1} b_{2}-2 a_{2} b_{1}, 2 a_{3} b_{1}-2 a_{1} b_{3}, a_{2} b_{6}\right.} \\
-a_{6} b_{2}+a_{5} b_{3}-a_{3} b_{5}+a_{5} b_{6}-a_{6} b_{5}, 2 a_{1} b_{5} \\
-2 a_{5} b_{1}+2 a_{4} b_{2}-2 a_{2} b_{4}+2 a_{4} b_{5} \\
-2 a_{5} b_{4}, 2 a_{3} b_{4}-2 a_{4} b_{3}+2 a_{6} b_{1} \\
\left.-2 a_{1} b_{6}+2 a_{6} b_{4}-2 a_{4} b_{6}\right) .
\end{aligned}
$$


Thus, under the Lie algebra $R^{6}$, the Lax pair (36) can be written as

$$
\begin{gathered}
U=\left(-\lambda, u \lambda, v, 0, u_{1}, \lambda, u_{2}\right)^{T}, \\
V=\left(V_{1} \lambda, V_{2} \lambda, V_{3}, V_{4} \lambda, V_{5} \lambda, V_{6}\right)^{T} .
\end{gathered}
$$

In terms of (48) and (49) we obtain that

$$
\begin{gathered}
\left\{V, \frac{\partial U}{\partial u}\right\}=\left(\eta_{1} V_{3}+\eta_{2} V_{6}\right) \lambda, \\
\left\{V, \frac{\partial U}{\partial v}\right\}=\left(\eta_{1} V_{2}+\eta_{2} V_{5}\right) \lambda, \\
\left\{V, \frac{\partial U}{\partial u_{1}}\right\}=\left(\eta_{2} V_{3}+\eta_{2} V_{6}\right) \lambda, \\
\left\{V, \frac{\partial U}{\partial u_{2}}\right\}=\left(\eta_{2} V_{2}+\eta_{2} V_{5}\right) \lambda, \\
\left\{V, \frac{\partial U}{\partial \lambda}\right\}=-2 \eta_{1} V_{1}+\left(\eta_{1} u+\eta_{2} u_{1}\right) V_{3} \\
-2 \eta_{2} V_{4}+\left(\eta_{2} u+\eta_{2} u_{1}\right) V_{6} .
\end{gathered}
$$

Substituting the above results into the variational identity yields

$$
\begin{gathered}
\frac{\delta}{\delta w} \int^{x}\left(-2 \eta_{1} V_{1}+\left(\eta_{1} u+\eta_{2} u_{1}\right) V_{3}+\eta_{2}\left(u+u_{1}\right) V_{6}\right) d x \\
=\lambda^{-\gamma} \frac{\partial}{\partial \lambda} \lambda^{\gamma}\left(\begin{array}{c}
\left(\eta_{1} V_{3}+\eta_{2} V_{6}\right) \lambda \\
\left(\eta_{1} V_{2}+\eta_{2} V_{5}\right) \lambda \\
\left(\eta_{2} V_{3}+\eta_{2} V_{6}\right) \lambda \\
\left(\eta_{2} V_{2}+\eta_{2} V_{5}\right) \lambda
\end{array}\right),
\end{gathered}
$$

where

$$
\frac{\delta}{\delta w}=\left(\frac{\delta}{\delta u}, \frac{\delta}{\delta v}, \frac{\delta}{\delta u_{1}}, \frac{\delta}{\delta u_{2}}\right)^{T} .
$$

Comparing the coefficients of $\lambda^{-n}$ on both sides in (51) gives

$$
\begin{gathered}
\frac{\delta}{\delta w} \int^{x}\left(-2 \eta_{1} V_{1, n+1}+\left(\eta_{1} u+\eta_{2} u_{1}\right) V_{3, n+1}\right. \\
\left.+2 \eta_{2} V_{4, n+1}+\eta_{2}\left(u+u_{1}\right) V_{6, n+1}\right) d x \\
=(-n+1+\gamma)\left(\begin{array}{l}
\eta_{1} V_{3 n}+\eta_{2} V_{6 n} \\
\eta_{1} V_{2 n}+\eta_{2} V_{5 n} \\
\eta_{2} V_{3 n}+\eta_{2} V_{6 n} \\
\eta_{2} V_{2 n}+\eta_{2} V_{5 n}
\end{array}\right) .
\end{gathered}
$$

From (37) we have $\gamma=-1$. Thus, we get that

$$
\left(\begin{array}{l}
\eta_{1} V_{3 n}+\eta_{2} V_{6 n} \\
\eta_{1} V_{2 n}+\eta_{2} V_{5 n} \\
\eta_{2} V_{3 n}+\eta_{2} V_{6 n} \\
\eta_{2} V_{2 n}+\eta_{2} V_{5 n}
\end{array}\right)=\frac{\delta H_{n+1}}{\delta u}
$$

where

$$
\begin{aligned}
H_{n+1}=\int_{1 / n}^{x}( & 2 \eta_{1} V_{1, n+1}-\left(\eta_{1} u+\eta_{2} u_{1}\right) V_{3, n+1} \\
& \left.+2 \eta_{2} V_{4, n+1}-\eta_{2}\left(u+u_{1}\right) V_{6, n+1}\right) d x .
\end{aligned}
$$

Therefore, we obtain the Hamiltonian structure of the nonlinear integrable coupling (44) as follows:

$$
\begin{aligned}
& \frac{\delta}{\delta w} \int^{x}\left(-2 \eta_{1} V_{1, n+1}+\left(\eta_{1} u+\eta_{2} u_{1}\right) V_{3, n+1}\right. \\
& \left.-2 \eta_{2} V_{4, n+1}+\eta_{2}\left(u+u_{1}\right) V_{6, n+1}\right) d x, \\
& W_{t_{n}}=\left(\begin{array}{c}
u \\
v \\
u_{1} \\
u_{2}
\end{array}\right)_{t_{n}} \\
& =\left(\begin{array}{cccc}
0 & \frac{-\partial}{\eta_{1}-\eta_{2}} & 0 & \frac{\partial}{\eta_{1}-\eta_{2}} \\
\frac{-\partial}{\eta_{1}-\eta_{2}} & 0 & \frac{\partial}{\eta_{1}-\eta_{2}} & 0 \\
0 & \frac{\partial}{\eta_{1}-\eta_{2}} & 0 & \frac{\eta_{1} \partial}{\left(\eta_{1}-\eta_{2}\right) \eta_{2}} \\
\frac{\partial}{\eta_{1}-\eta_{2}} & 0 & \frac{\eta_{1} \partial}{\left(\eta_{1}-\eta_{2}\right) \eta_{2}} & 0
\end{array}\right) \\
& \times\left(\begin{array}{l}
\eta_{1} V_{3 n}+\eta_{2} V_{6 n} \\
\eta_{1} V_{2 n}+\eta_{2} V_{5 n} \\
\eta_{2} V_{3 n}+\eta_{2} V_{6 n} \\
\eta_{2} V_{2 n}+\eta_{2} V_{5 n}
\end{array}\right) \\
& =J\left(\begin{array}{l}
\eta_{1} V_{3 n}+\eta_{2} V_{6 n} \\
\eta_{1} V_{2 n}+\eta_{2} V_{5 n} \\
\eta_{2} V_{3 n}+\eta_{2} V_{6 n} \\
\eta_{2} V_{2 n}+\eta_{2} V_{5 n}
\end{array}\right)=J \frac{\delta H_{n+1}}{\delta u},
\end{aligned}
$$

where $J$ is obviously Hamiltonian.

\section{The Second Expanding Integrable Model of the GC Hierarchy}

In this section we construct a new 6-dimensional Lie algebra to discuss the second integrable coupling of the GC hierarchy. Set

$$
\begin{gathered}
h_{1}=f_{1}, \quad h_{2}=f_{2}, \quad h_{3}=f_{3}, \\
h_{j}=\left(\begin{array}{cc}
0 & e_{j-3} \\
e_{j-3} & 0
\end{array}\right), \quad j=4,5,6 .
\end{gathered}
$$

It is easy to see that

$$
\begin{array}{rrrr}
{\left[h_{1}, h_{2}\right]=2 h_{2},} & {\left[h_{1}, h_{3}\right]=-2 h_{3},} & {\left[h_{2}, h_{3}\right]=h_{1},} \\
{\left[h_{1}, h_{4}\right]=0,} & {\left[h_{1}, h_{5}\right]=2 h_{5},} & {\left[h_{1}, h_{6}\right]=-2 h_{6},} \\
{\left[h_{2}, h_{4}\right]=-2 h_{5},} & {\left[h_{2}, h_{5}\right]=0,} & {\left[h_{2}, h_{6}\right]=h_{4},} \\
{\left[h_{3}, h_{4}\right]=2 h_{6},} & {\left[h_{3}, h_{5}\right]=-h_{4},} & {\left[h_{3}, h_{6}\right]=0,} \\
{\left[h_{4}, h_{5}\right]=2 h_{2},} & {\left[h_{4}, h_{6}\right]=-2 h_{3},} & {\left[h_{5}, h_{6}\right]=h_{1} .}
\end{array}
$$


If we set $G=\operatorname{span}\left\{h_{1}, \ldots, h_{6}\right\}, G_{1}=\operatorname{span}\left\{h_{1}, h_{2}, h_{3}\right\}$, and $G_{2}=$ $\operatorname{span}\left\{h_{4}, h_{5}, h_{6}\right\}$, then we have that

$$
G=G_{1}+G_{2}, \quad\left[G_{1}, G_{2}\right] \text { not in } G_{2} .
$$

Hence, the integrable couplings of the GC hierarchy cannot be generated by the Lie algebra $G$ as above under the frame of the Tu scheme. In what follows, we will deduce a nonlinear expanding integrable model of the GC hierarchy.

Set

$$
\begin{gathered}
U=-h_{1}(1)+u h_{2}(1)+v h_{3}(0)+w_{1} h_{5}(1)+w_{2} h_{6}(0) \\
V=\sum_{m \geq 0}\left(V_{1 m} h_{1}(1-m)+V_{2 m} h_{2}(1-m)+V_{3 m} h_{3}(-m)\right. \\
+V_{4 m} h_{4}(1-m)+V_{5 m} h_{5}(1-m) \\
\left.+V_{6 m} h_{6}(1-m)\right)
\end{gathered}
$$

where $h_{i}(m)=h_{i} \lambda^{m}, i=1,2,3,4,5,6$.

Solving the stationary zero curvature equation

$$
V_{x}=[U, V]
$$

gives rise to

$$
\begin{gathered}
\left(V_{1 m}\right)_{x}=u V_{3 m}-v V_{2 m}-w_{2} V_{5 m}+w_{1} V_{6 m} \\
\left(V_{2 m}\right)_{x}=-2 V_{2, m+1}-2 u V_{1, m+1}-2 w_{1} V_{4, m+1} \\
\left(V_{3 m}\right)_{x}=2 V_{3, m+1}+2 v V_{1, m+1}+2 w_{2} V_{4, m+1} \\
\left(V_{4 m}\right)_{x}=u V_{6 m}-v V_{5 m}+w_{1} V_{3 m}-w_{2} V_{2 m} \\
\left(V_{5 m}\right)_{x}=-2 V_{5, m+1}-2 u V_{4, m+1}-2 w_{1} V_{1, m+1} \\
\left(V_{6 m}\right)_{x}=2 V_{6, m+1}+2 v V_{4, m+1}+2 w_{2} V_{1, m+1}
\end{gathered}
$$

Let $V_{2,1}=-\alpha u, V_{3,1}=-\alpha v, V_{5,1}=-\alpha w_{1}$, and $V_{6,1}=\alpha w_{2}$; then one gets from (62) that

$$
\begin{gathered}
V_{1,2}=-\frac{\alpha}{2} u v, \quad V_{2,2}=\frac{\alpha}{2}\left(u_{x}+u^{2} v\right), \\
V_{3,2}=\frac{\alpha}{2}\left(-v_{x}+u v^{2}\right), \quad V_{4,1}=0 \\
V_{4,2}=\frac{\alpha}{2}\left(u w_{2}-w_{1} v\right), \quad V_{5,2}=\frac{\alpha}{2}\left(w_{1, x}-u^{2} w_{2}+w_{1} u v\right), \\
V_{6,2}=\frac{\alpha}{2}\left(w_{2, x}+v^{2} w_{1}\right), \ldots
\end{gathered}
$$

Noting $V_{+}^{(n)}=\sum_{m=0}^{n}\left(V_{1 m} h_{1}(1+n-m)+V_{2 m} h_{2}(1+n-m)+\right.$ $V_{3 m} h_{3}(n-m)+V_{4 m} h_{4}(1+n-m)+V_{5 m} h_{5}(1+n-m)+V_{6 m} h_{6}(n-$ $m))=\lambda^{n} V-V_{-}^{(n)}$, one infers that $-V_{+, x}^{(n)}+\left[U, V_{+}^{(n)}\right]=\left(2 V_{2, n+1}+\right.$ $\left.2 u V_{1, n+1}+2 w_{1} V_{4, n+1}\right) h_{2}(1)-2\left(V_{3, n+1}+v V_{1, n+1}+w_{2} V_{4, n+1}\right) h_{3}(0)+$ $\left(2 V_{5, n+1}+2 u V_{4, n+1}+2 w_{1} V_{1, n+1}\right) h_{5}(1)-2\left(V_{6, n+1}+v V_{4, n+1}+\right.$ $\left.w_{2} V_{1, n+1}\right) h_{6}(0)$.

Set $V^{(n)}=V_{+}^{(n)}$, by employing the zero curvature equation

$$
U_{t_{n}}-V_{x}^{(n)}+\left[U, V^{(n)}\right]=0
$$

we have

$$
\bar{u}_{t_{n}}=\left(\begin{array}{c}
u \\
v \\
w_{1} \\
w_{2}
\end{array}\right)_{t_{n}}=\left(\begin{array}{c}
-2 V_{2, n+1}-2 u V_{1, n+1}-2 w_{1} V_{4, n+1} \\
2 V_{3, n+1}+2 v V_{1, n+1}+2 w_{2} V_{4, n+1} \\
-2 V_{5, n+1}-2 u V_{4, n+1}-2 w_{1} V_{1, n+1} \\
2 V_{6, n+1}+2 v V_{4, n+1}+2 w_{2} V_{1, n+1}
\end{array}\right)
$$

When $n=2, \alpha=2,(65)$ reduces to

$$
\begin{gathered}
u_{t_{2}}=u_{x x}+\left(u^{2} v\right)_{x}, \quad v_{t_{2}}=-v_{x x}+\left(u v^{2}\right)_{x} \\
w_{1, t_{2}}=w_{1, x x}-\left(u^{2} w_{2}\right)_{x}+\left(w_{1} u v\right)_{x} \\
w_{2, t_{2}}=w_{2, x x}+\left(v^{2} w_{1}\right)_{x} .
\end{gathered}
$$

It is remarkable that (66) is linear with respect to the variables $w_{1}, w_{2}$; however, it is nonlinear.

Equation (60) can be written as

$$
\begin{aligned}
U & =\left(\begin{array}{cccc}
-\lambda & u \lambda & 0 & w_{1} \lambda \\
v & \lambda & w_{2} & 0 \\
0 & w_{1} \lambda & -\lambda & u \lambda \\
w_{2} & 0 & v & \lambda
\end{array}\right), \\
V & =\left(\begin{array}{cccc}
V_{1} \lambda & V_{2} \lambda & V_{4} \lambda & V_{5} \lambda \\
V_{3} & -V_{1} \lambda & V_{6} & -V_{4} \lambda \\
V_{4} \lambda & V_{5} \lambda & V_{1} \lambda & V_{2} \lambda \\
V_{6} & -V_{4} \lambda & V_{3} & -V_{1} \lambda
\end{array}\right) .
\end{aligned}
$$

By computing that

$$
\begin{gathered}
\frac{\partial U}{\partial u}=\left(\begin{array}{llll}
0 & \lambda & 0 & 0 \\
0 & 0 & 0 & 0 \\
0 & 0 & 0 & \lambda \\
0 & 0 & 0 & 0
\end{array}\right), \quad \frac{\partial U}{\partial v}=\left(\begin{array}{llll}
0 & 0 & 0 & 0 \\
1 & 0 & 0 & 0 \\
0 & 0 & 0 & 0 \\
0 & 0 & 1 & 0
\end{array}\right), \\
\frac{\partial U}{\partial w_{1}}=\left(\begin{array}{llll}
0 & 0 & 0 & \lambda \\
0 & 0 & 0 & 0 \\
0 & \lambda & 0 & 0 \\
0 & 0 & 0 & 0
\end{array}\right), \\
\left.\frac{\partial U}{\partial \lambda}=\left(\begin{array}{llll}
0 & 0 & 0 & 0 \\
0 & 0 & 1 & 0 \\
0 & 0 & 0 & 0 \\
1 & 0 & 0 & 0
\end{array}\right), \quad \begin{array}{cccc}
-1 & u & 0 & w_{1} \\
0 & 1 & 0 & 0 \\
0 & w_{1} & -1 & u \\
0 & 0 & 0 & 1
\end{array}\right),
\end{gathered}
$$

thus, we have

$$
\begin{gathered}
\left\langle V, \frac{\partial U}{\partial u}\right\rangle=2 \lambda V_{3}, \quad\left\langle V, \frac{\partial U}{\partial v}\right\rangle=2 \lambda V_{2}, \\
\left\langle V, \frac{\partial U}{\partial w_{1}}\right\rangle=2 \lambda V_{6}, \quad\left\langle V, \frac{\partial U}{\partial w_{2}}\right\rangle=2 \lambda V_{5}, \\
\left\langle V, \frac{\partial U}{\partial \lambda}\right\rangle=-4 \lambda V_{1}+2 u V_{3}+2 w_{1} V_{6} .
\end{gathered}
$$


Substituting the above consequences into the trace identity proposed by $\mathrm{Tu}[14]$ yields that

$$
\frac{\delta}{\delta \bar{u}}\left(-4 \lambda V_{1}+2 u V_{3}+2 w_{1} V_{6}\right)=\lambda^{-\gamma} \frac{\partial}{\partial \lambda} \lambda^{\gamma}\left(\begin{array}{c}
2 \lambda V_{3} \\
2 \lambda V_{2} \\
2 \lambda V_{6} \\
2 \lambda V_{5}
\end{array}\right)
$$

Comparing the coefficients of $\lambda^{-n}$ gives

$$
\begin{gathered}
\frac{\delta}{\delta \bar{u}}\left(-4 V_{1, n}+2 u V_{3, n-1}+2 w_{1} V_{6, n-1}\right) \\
=(-n+1+\gamma)\left(\begin{array}{l}
2 V_{3 n} \\
2 V_{2 n} \\
2 V_{6 n} \\
2 V_{5 n}
\end{array}\right) .
\end{gathered}
$$

Inserting the initial values in (62) gives $\gamma=-1$. Therefore, we obtain that

$$
\left(\begin{array}{l}
2 V_{3 n} \\
2 V_{2 n} \\
2 V_{6 n} \\
2 V_{5 n}
\end{array}\right)=\frac{\delta}{\delta \bar{u}}\left(\frac{4 V_{1 n}-2 u V_{3, n-1}-2 w_{1} V_{6, n-1}}{n}\right) \equiv \frac{\delta H_{n}}{\delta \bar{u}},
$$

where $H_{n}=(1 / n)\left(4 V_{1 n}-2 u V_{3, n-1}-2 w_{1} V_{6, n-1}\right)$ are conserved densities of the expanding integrable model (65). Thus, (65) can be written as the Hamiltonian structure

$$
\bar{u}_{t_{n}}=\left(\begin{array}{c}
u \\
v \\
w_{1} \\
w_{2}
\end{array}\right)_{t_{n}}=J \frac{\delta H_{n}}{\delta \bar{u}}
$$

where $J=(1 / 2)\left(\begin{array}{llll}0 & \partial & 0 & 0 \\ \partial & 0 & 0 & 0 \\ 0 & 0 & 0 & \partial \\ 0 & 0 & \partial & 0\end{array}\right), \partial=\partial / \partial x$, is a Hamiltonian operator.

\section{Conflict of Interests}

The authors declare that there is no conflict of interests regarding the publication of this paper.

\section{Acknowledgments}

This work was supported by the Natural Science Foundation of China (11371361) and the Natural Science Foundation of Shandong Province (ZR2012AQ011, ZR2012AQ015).

\section{References}

[1] W. X. Ma and B. Fuchssteiner, "Integrable theory of the perturbation equations," Chaos, Solitons and Fractals, vol. 7, no. 8, pp. 1227-1250, 1996.

[2] W.-X. Ma, "Integrable couplings of soliton equations by perturbations-I. A general theory and application to the KdV hierarchy," Methods and Applications of Analysis, vol. 7, no. 1, pp. 21-55, 2000.
[3] W.-X. Ma, X.-X. Xu, and Y. Zhang, "Semi-direct sums of Lie algebras and continuous integrable couplings," Physics Letters A, vol. 351, no. 3, pp. 125-130, 2006.

[4] Y. Zhang, "A generalized multi-component Glachette-Johnson (GJ) hierarchy and its integrable coupling system," Chaos, Solitons and Fractals, vol. 21, no. 2, pp. 305-310, 2004.

[5] Y. F. Zhang and H. Q. Zhang, "A direct method for integrable couplings of TD hierarchy," Journal of Mathematical Physics, vol. 43, no. 1, pp. 466-472, 2002.

[6] T. C. Xia and F.C. You, "Multi-component Dirac equation hierarchy and its multi-component integrable couplings system," Chinese Physics B, vol. 16, no. 3, p. 605, 2007.

[7] Y. F. Zhang and F. K. Guo, "Matrix Lie algebras and integrable couplings," Communications in Theoretical Physics, vol. 46, no. 5, pp. 812-818, 2006.

[8] E. Fan and Y. Zhang, "A simple method for generating integrable hierarchies with multi-potential functions," Chaos, Solitons and Fractals, vol. 25, no. 2, pp. 425-439, 2005.

[9] W.-X. Ma and Z.-N. Zhu, "Constructing nonlinear discrete integrable Hamiltonian couplings," Computers \& Mathematics with Applications, vol. 60, no. 9, pp. 2601-2608, 2010.

[10] Y. F. Zhang, "Lie algebras for constructing nonlinear integrable couplings," Communications in Theoretical Physics, vol. 56, p. 805, 2011.

[11] Y. F. Zhang and Y. C. Hon, "Some evolution hierarchies derived from self-dual Yang-Mills equations," Communications in Theoretical Physics, vol. 56, p. 856, 2011.

[12] H. Y. Wei and T.C. Xia, "Nonlinear integrable couplings of super Kaup-Newell hierarchy and its super Hamiltonian structures," Acta Physica Sinica, vol. 62, Article ID 120202, 2013.

[13] X. G. Geng and C. W. Cao, "Quasi-periodic solutions of the $2+$ 1 dimensional modified Korteweg-de Vries equation," Physics Letters A, vol. 261, no. 5-6, pp. 289-296, 1999.

[14] G. Z. Tu, "The trace identity, a powerful tool for constructing the Hamiltonian structure of integrable systems," Journal of Mathematical Physics, vol. 30, no. 2, pp. 330-338, 1989.

[15] W.-X. Ma and M. Chen, "Hamiltonian and quasi-Hamiltonian structures associated with semi-direct sums of Lie algebras," Journal of Physics A, vol. 39, no. 34, pp. 10787-10801, 2006.

[16] Y.-F. Zhang and H. Tam, "A few new higher-dimensional Lie algebras and two types of coupling integrable couplings of the AKNS hierarchy and the KN hierarchy," Communications in Nonlinear Science and Numerical Simulation, vol. 16, no. 1, pp. 76-85, 2011. 


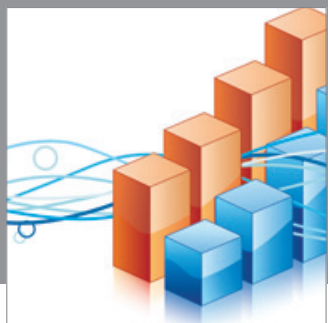

Advances in

Operations Research

mansans

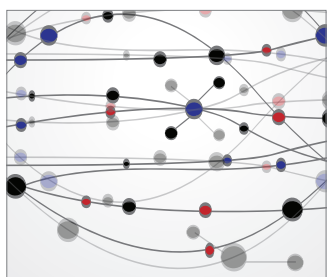

The Scientific World Journal
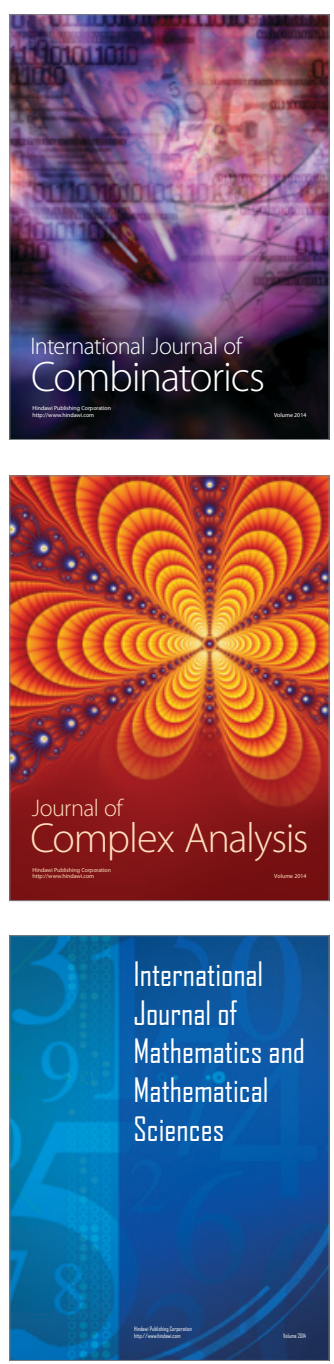
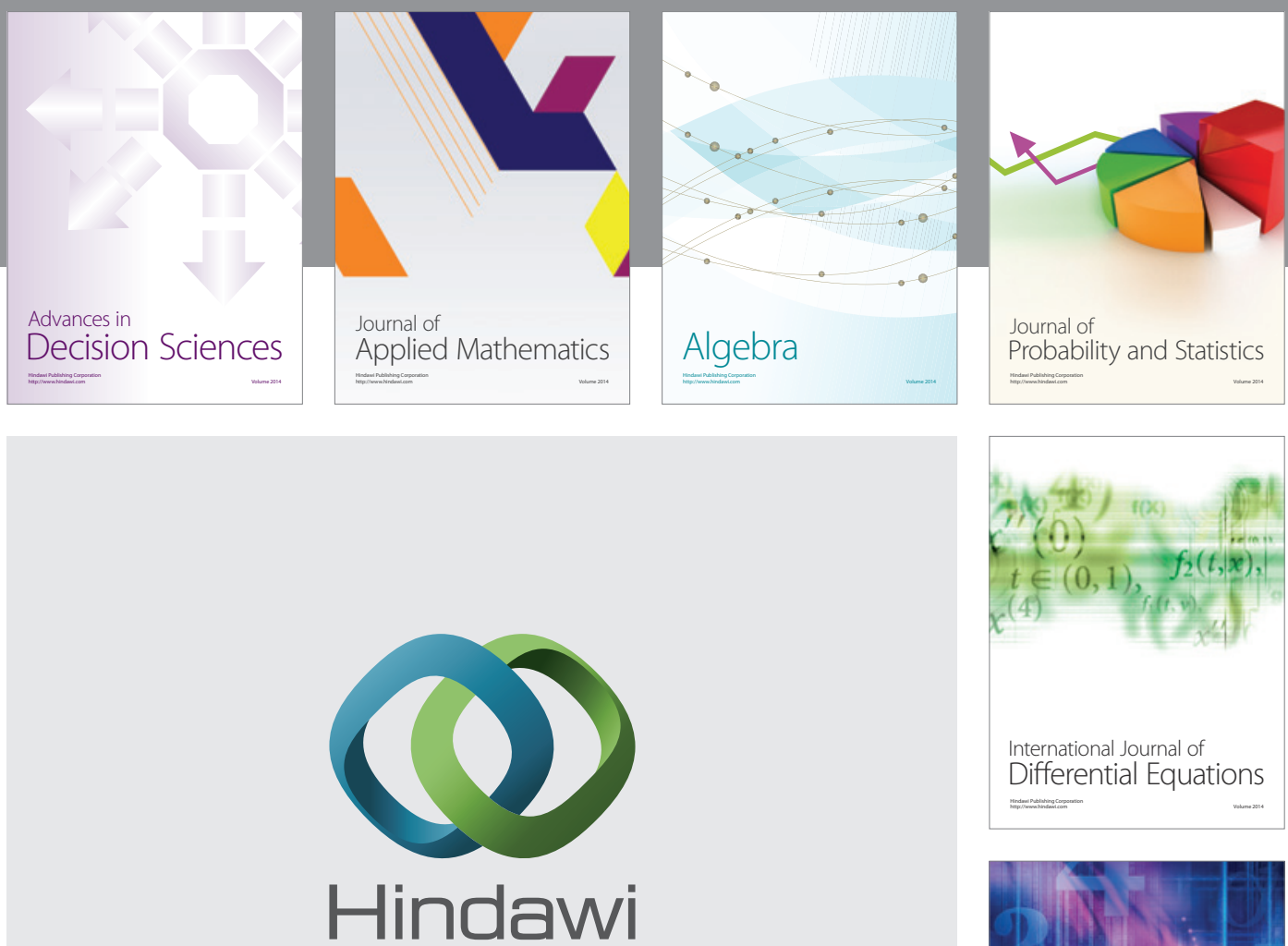

Submit your manuscripts at http://www.hindawi.com
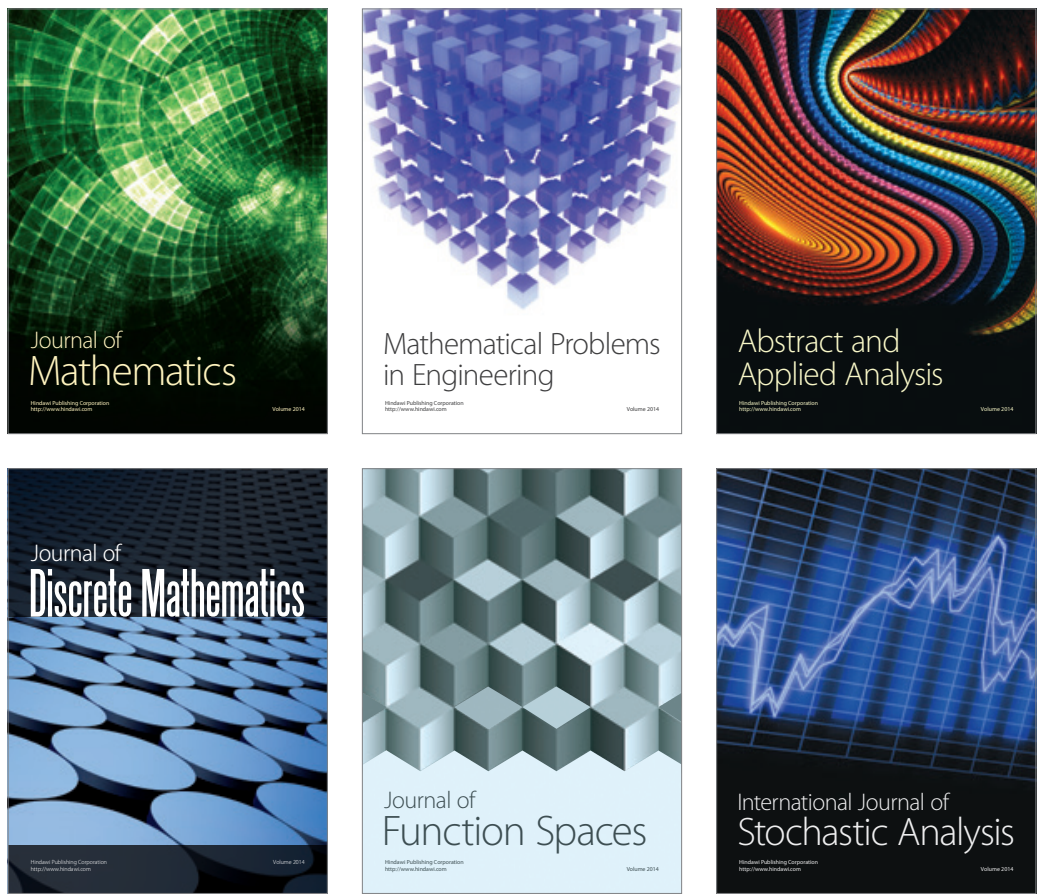

Journal of

Function Spaces

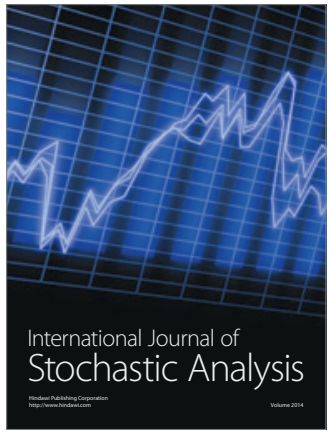

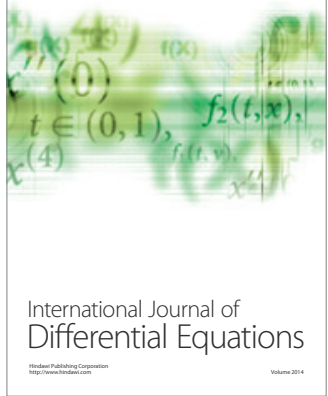
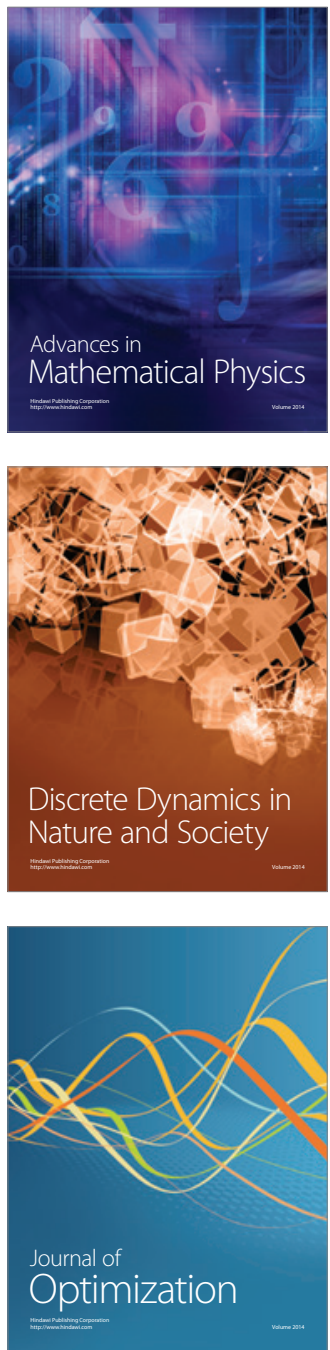